Editorial

\title{
This special issue of Nuclear Engineering and Design collects selected papers from the "specialists workshop on advanced instrumentation and measurement techniques for nuclear reactor thermal-hydraulics" (SWINTH), held in Livorno, Italy, on June 2016
}

The workshop was conceived and organized by SILENCE ("Significant Light and Heavy Water Reactor Thermal Hydraulic Experiments Network for the Consistent Exploitation of the Data"), a Network of Companies and Research Organizations that are concerned with - and active in the area of - nuclear reactor thermal-hydraulic experimentation to support safety assessment and design of watercooled nuclear reactors. SILENCE Network was formally constituted in 2014 by AREVA GmbH (Germany; now Framatome), the Helmholtz Zentrum Dresden Rossendorf (HZDR, Germany), the Korean Atomic Energy Research Institute (KAERI, Korea), the Lappeenranta University of Technology (LUT, Finland), the Hungarian Academy of Science Centre for Energy Research (MTA EK, Hungary) and the Paul Scherrer Institute (PSI, Switzerland), with the San Piero a Grado Nuclear Research Group (GRNSPG, Italy) of the University of Pisa playing the role of Host Organization, and with administrative and managerial support from NINE - Nuclear and Industrial Engineering. More recently also the Commissariat à l'Energie Atomique et aux Energies Alternatives (CEA, France), Becker Technologies GmbH (Germany) and the State Power Investment Corporation Research Institute (SPICRI, China) have joined the Network.

Significant advances have been accomplished in the instrumentation for two-phase flow since the "OECD/NEA/CSNI Specialists Meeting on Advanced Instrumentation and Measurement Techniques" held in Santa-Barbara, California, US, on March 17-20, 1997. The idea of organizing a new international workshop on instrumentation and measurement techniques emerged during SILENCE meetings. It is consistent with both the "vision" and the "mission" of SILENCE Network, which promotes and fosters the establishment of a common ground for cooperation and discussion on thermal-hydraulic experiments, and wants to promote new experiments, including improvements of the existing measurement techniques.

The purpose of SWINTH was to bring together international experts on instrumentation, experiments and modelling in order to:

- review the recent instrumentation and experiment techniques developments;

- identify the specific experimental needs that arose from the development of modern simulation tools including system codes, component codes, and computational multi-fluid dynamics (CMFD) codes provided with advanced models such as dynamic interfacial area modelling, poly-dispersion modelling of bubbly and droplet flow, multi-field models and two-phase turbulence models;

- discuss future directions for instrumentation developments, modelling and experiments.

The subject is wide and complex and deserves "dedicated" discussion; therefore, specialized workshops such as the present one can be complementary to other events on code development and verification and validation $(\mathrm{V} \& \mathrm{~V})$ and initiatives in which the experimental area is not covered with sufficient detail and focus.

The following topics were included in the scope of the workshop:

- modelling needs for closure relations in current and advanced 1D models and in 3D models for either porous medium or open medium approaches;

- local instrumentation for void fraction, interfacial area, bubble and droplet size, phase velocities, turbulence scales, mean and fluctuating temperature field, mean and fluctuating pressure, non-condensable gas fraction, wall friction, wall heat flux (this may include optical probes, conductivity or capacity probes, film probes, Wire Mesh Sensors, hot wire or hot film anemometry, steady and transient X-ray or Gamma tomography, absorption and scattering methods, Laser Doppler Anemometry, Particle Tracking Velocimetry, Laser Induced Fluorescence, Nuclear Magnetic Resonance, ultrasonic methods, and any other new advanced method);

- visualization techniques, expert systems, identification of flow regimes in complex geometry (rod bundle, tube bundle, etc.) and/or in high pressure and high temperature conditions;

- use of simulant fluids with well-established similarity laws;

- any new experimental technique;

- industrially applicable instrumentation.

The workshop was also meant to address quality- and qualificationrelated aspects, such as:

- current code validation requirements;

- test design requirements for code validation (e.g. pressure losses, scaling issues, etc.);

- specific requirements for CMFD-grade experiments and related 
measurements for single- and multi-phase flows;

- criteria for quality of data (e.g. measurement uncertainty assessment);

- experimental data handling issues.

The workshop was expected (and actually succeeded) to help identifying the current gaps between the (modelling and code qualification) needs and the available technology, and the margins for future advancements. SWINTH also constituted an opportunity for networking between experimentalists and scientists operating on the code development and validation side.

About fifty abstracts were initially submitted to the Organizing Committee upon workshop announcement. Eventually, thirty-eight papers were selected for oral presentation, after a rigorous review process by the SWINTH Scientific Committee. The workshop involved plenary sessions only, in order to give all participants the chance to attend all technical presentations, as well as four keynote lectures given by internationally acknowledged top-level experts. The workshop was attended by sixty-five people, most of whom - this is at least the organizers' feeling! - appreciated the high quality of the technical contributions (many of which focused on innovative solutions and state-ofthe-art applications) and of the discussions originated therein. Also, many expressed the wish that further SWINTH workshops be organized in the future. In this connection, we can now announce that a new SWINTH will be held in 2019 , and this time with a key organizing role by the OECD/NEA/CSNI Working Group on Accident Management and Analysis (WGAMA), together with the SILENCE Network.

The papers included in this special issue were selected by the Scientific Committee upon indications from session chairpersons, and then went through a further review and revision process according to NED standards. Other SWINTH papers, which might have deserved being included, followed different editorial paths. The result is anyway a good sample of the workshop contents, and the SWINTH organizers deeply appreciate this opportunity to share it with NED's readers and to promote the SWINTH initiatives!

The Guest Editors Nusret Aksan Switzerland

Dominique Bestion CEA, France

Francesco D'Auria Univ. of Pisa, Italy

Yassin Hassan

Texas A\&M Univ., USA

Fabio Moretti NINE, Italy

E-mail address: f.moretti@nineeng.com 